TRANSACTIONS OF THE

AMERICAN MATHEMATICAL SOCIETY

Volume 355, Number 8 , Pages 3047-3064

S 0002-9947(01)02893-8

Article electronically published on September 21, 2001

\title{
MODERATE DEVIATION PRINCIPLES FOR TRAJECTORIES OF SUMS OF INDEPENDENT BANACH SPACE VALUED RANDOM VARIABLES
}

\author{
YIJUN HU AND TZONG-YOW LEE
}

\begin{abstract}
Let $\left\{X_{n}\right\}$ be a sequence of i.i.d. random vectors with values in a separable Banach space. Moderate deviation principles for trajectories of sums of $\left\{X_{n}\right\}$ are proved, which generalize related results of Borovkov and Mogulskii (1980) and Deshayes and Picard (1979). As an application, functional laws of the iterated logarithm are given. The paper also contains concluding remarks, with examples, on extending results for partial sums to corresponding ones for trajectory setting.
\end{abstract}

\section{INTRODUCTION AND MAIN RESULTS}

Let $\left\{X_{n}\right\}$ be a sequence of i.i.d. $\mathbf{R}^{d}$-valued random variables, satisfying $E X_{1}=0$ and $\operatorname{Var}\left(X_{1}\right)<+\infty$. Let $\widetilde{S}_{n}$ be the trajectories of sums of $\left\{X_{n}\right\}$, that is, $\widetilde{S}_{n}(t)=$ $\sum_{i=1}^{[n t]} X_{i}+(n t-[n t]) X_{[n t]+1}, t \in[0,1]$. Deshayes and Picard [19] have studied moderate deviations (MDs) for $\left\{\widetilde{S}_{n}\right\}$ in $C[0,1]$, which generalized corresponding results obtained by Borovkov [10] and Mogulskii [30. Borovkov and Mogulskii [12] extended Deshayes and Picard's 19] results to independent, identically distributed (i.i.d.) random vectors $\left\{X_{n}\right\}$ with values in a complete locally convex Hausdorff topological vector space, under the crucial assumption that

$$
\mu \triangleq \mathcal{L}\left(X_{1}\right) \in \mathrm{CLT} .
$$

That is, the law $\mathcal{L}\left(\frac{1}{\sqrt{n}} \sum_{i=1}^{n} X_{i}\right)$ converges weakly to a non-degenerate normal distribution. For more related results, see Borovkov and Mogulskii 12 and the references therein. However, the CLT for i.i.d. random vectors $\left\{X_{n}\right\}$ is not easily satisfied when $\left\{X_{n}\right\}$ take values in a general Banach space. Motivated by the observation above, in the present paper, we shall investigate the moderate deviation principle (MDP) for the trajectories, $\widetilde{S}_{n}$, of sums of i.i.d. random vectors $\left\{X_{n}\right\}$ with values in a separable Banach space, and aim at removing the assumption employed by

Received by the editors March 28, 2001 and, in revised form, May 3, 2001.

2000 Mathematics Subject Classification. Primary 60F10.

Key words and phrases. Moderate deviations, trajectories, functional law of the iterated logarithm.

Supported in part by the National Natural Science Foundation of China and the Education Department of China. 
Borovkov and Mogulskii [12, $\mu \triangleq \mathcal{L}\left(X_{1}\right) \in$ CLT. As an application, the functional laws of the iterated logarithm are given.

Now, we turn to describing our main results in detail. Let $\mathbf{N}$ be the set of positive integers. For $x \in \mathbf{R},[x]$ denotes the greatest integer $k \leq x$. Throughout this paper, $(\mathbf{E},\|\cdot\|)$ will denote a separable Banach space and $\mathbf{E}^{*}$ its dual space. For $N \in \mathbf{N}$, we endow the product space $\mathbf{E}^{N}$ with the product topology, which can be induced by the metric $d(\cdot, \cdot)$ given by $d(x, y)=\sum_{i=1}^{N}\left\|x_{i}-y_{i}\right\|, x=$ $\left(x_{1}, \cdots, x_{N}\right), y=\left(y_{1}, \cdots, y_{N}\right) \in \mathbf{E}^{N}$. Let $\left\{X_{n}\right\}$ be a sequence of independent $\mathbf{E}$ valued random vectors with common distribution $\mu$ such that $\mu \in W M_{0}^{2}$, that is, $E f\left(X_{1}\right)=0$ and $E f^{2}\left(X_{1}\right)<\infty$ for every $f \in \mathbf{E}^{*}$. Let $\left(H,\|\cdot\|_{H}\right)$ be the reproducing kernel Hilbert space associated to $\mu$ (see Goodman, Kuelbs and Zinn 22]). A good example, which reveals the structure, is the Wiener measure $\mu$ on $\mathbf{E}=C[0,1]$ with the supremum norm. Then the associated reproducing kernel Hilbert space is the so-called Cameron-Martin space. Let $S_{n}=\sum_{i=1}^{n} X_{i}, S_{0}=0$. We denote by $\widetilde{S}_{n}$ the trajectories of sums of $\left\{X_{n}\right\}$. In other words,

$$
\widetilde{S}_{n}(t)=S_{[n t]}+(n t-[n t]) X_{[n t]+1}, \quad t \in[0,1] .
$$

Denote by $S_{n}(\cdot)$ the piecewise constant functions of sums of $\left\{X_{n}\right\}$, that is,

$$
S_{n}(t)=\sum_{i=1}^{[n t]} X_{i}, \quad t \in[0,1] .
$$

Let $C([0,1], \mathbf{E})$ be the Banach space of all continuous mappings from $[0,1]$ into $\mathbf{E}$ equipped with the sup-norm, $\|\cdot\|_{\infty}$. Denote by $D([0,1], \mathbf{E})$ the Banach space of all right-continuous mappings with left limits from $[0,1]$ into $\mathbf{E}$ equipped with the metric $d_{\infty}(f, g)=\sup _{0 \leq t \leq 1}\|f(t)-g(t)\|$. Given a set $A$, let $A^{c}$ and $\bar{A}$ stand for the complement and the closure of $A$, respectively.

Define a function $\Lambda: \mathbf{E} \rightarrow[0,+\infty]$

$$
\Lambda(x)= \begin{cases}\frac{1}{2}\|x\|_{H}^{2}, & \text { if } x \in H, \\ +\infty, & \text { otherwise }\end{cases}
$$

and define a mapping $\widetilde{\Lambda}: D([0,1], \mathbf{E}) \rightarrow[0,+\infty]$

$$
\widetilde{\Lambda}(f)= \begin{cases}\int_{0}^{1} \Lambda(g(s)) d s, & f \in \mathcal{A C}, \\ +\infty, & \text { otherwise }\end{cases}
$$

where $\mathcal{A C}=\left\{f \in D([0,1], \mathbf{E})\right.$; there exists $g \in L^{1}([0,1], \mathbf{E})$ such that $f(t)=$ $\int_{0}^{t} g(s) d s$ for $\left.t \in[0,1]\right\}$, and $L^{1}([0,1], \mathbf{E})$ is the space of $\mathbf{E}$-valued Bochner integrable functions on $[0,1]$. It is readily seen that $\mathcal{A C} \subset C([0,1], \mathbf{E})$.

Throughout this paper, for random vectors $\left\{Y_{n}\right\}$, we will write $Y_{n} \stackrel{P}{\longrightarrow} 0$ if $Y_{n} \rightarrow 0$ in probability as $n \rightarrow \infty$.

Throughout this paper, let $\{b(n) ; n \geq 1\}$ be a positive sequence such that

$$
\frac{b(n)}{\sqrt{n}} \rightarrow \infty, \quad \frac{b(n)}{n} \rightarrow 0 .
$$

The following conditions are assumed to be satisfied.

$$
E \exp \left(\beta\left\|X_{1}\right\|\right)<\infty \text { for some } \beta>0 \text {. }
$$




$$
\frac{S_{n}}{b(n)} \stackrel{P}{\longrightarrow} 0 \text {. }
$$

It is well-known that under conditions (1.4) and (1.5), $\left\{\frac{S_{n}}{b(n)}\right\}$ in $\mathbf{E}$ satisfies an MDP with speed $\left\{\frac{n}{b^{2}(n)}\right\}$ and the rate function $\Lambda$, defined by (1.1). By this we mean that, for every closed set $F \subset \mathbf{E}$,

$$
\limsup _{n \rightarrow \infty} \frac{n}{b^{2}(n)} \log P\left\{\frac{S_{n}}{b(n)} \in F\right\} \leq-\inf _{x \in F} \Lambda(x),
$$

and for every open set $G \subset \mathbf{E}$,

$$
\liminf _{n \rightarrow \infty} \frac{n}{b^{2}(n)} \log P\left\{\frac{S_{n}}{b(n)} \in G\right\} \geq-\inf _{x \in G} \Lambda(x) .
$$

Throughout this article, a rate function is understood to have compact level sets, i.e., $\{\Lambda \leq a\}$ is compact in $\mathbf{E}$ for all $a \geq 0$. For this result of the partial sum see Goodman, Kuelbs and Zinn [22, Lemma 2.1 (V)], Chen [13, Theorem 2]; [15. Theorem 1], de Acosta [2], Ledoux [26] and Jiang [24].

It should be mentioned that recently Arcones 7] gave necessary and sufficient conditions for MDP for $\left\{\frac{S_{n}}{b(n)}\right\}$ in the real-valued case.

The main results of this paper are following.

Theorem 1.1. Let $\pi: 0=t_{0}<t_{1}<\cdots<t_{N}=1$ be a partition of $[0,1]$. Assume (1.4) and (1.5) hold, then $\left\{\frac{1}{b(n)}\left(S_{n}\left(t_{1}\right), \cdots, S_{n}\left(t_{N}\right)\right)\right\}$ in $\mathbf{E}^{N}$ satisfies an MDP with speed $\left\{\frac{n}{b^{2}(n)}\right\}$ and a rate function $\widetilde{I}^{\pi}$, that is, for every closed set $F$ and open set $G$ of $\mathbf{E}^{N}$,

$$
\begin{aligned}
& \limsup _{n \rightarrow \infty} \frac{n}{b^{2}(n)} \log P\left\{\frac{1}{b(n)}\left(S_{n}\left(t_{1}\right), \cdots, S_{n}\left(t_{N}\right)\right) \in F\right\} \leq-\inf _{z \in F} \widetilde{I}^{\pi}(z), \\
& \liminf _{n \rightarrow \infty} \frac{n}{b^{2}(n)} \log P\left\{\frac{1}{b(n)}\left(S_{n}\left(t_{1}\right), \cdots, S_{n}\left(t_{N}\right)\right) \in G\right\} \geq-\inf _{z \in G} \widetilde{I}^{\pi}(z),
\end{aligned}
$$

where $\widetilde{I}^{\pi}: \mathbf{E}^{N} \rightarrow[0,+\infty]$ is given by

$$
\widetilde{I}^{\pi}(z)=\sum_{i=1}^{N}\left(t_{i}-t_{i-1}\right) \Lambda\left(\frac{z_{i}-z_{i-1}}{t_{i}-t_{i-1}}\right)
$$

for $z=\left(z_{1}, \cdots, z_{N}\right) \in \mathbf{E}^{N}, z_{0} \triangleq 0$.

So does $\left\{\frac{1}{b(n)}\left(\widetilde{S}_{n}\left(t_{1}\right), \cdots, \widetilde{S}_{n}\left(t_{N}\right)\right)\right\}$ in $\mathbf{E}^{N}$ with the same speed $\left\{\frac{n}{b^{2}(n)}\right\}$ and the same rate function $\widetilde{I}^{\pi}$.

Remark 1.1. It is readily checked that $\widetilde{I}^{\pi}$ inherits the property of compact level sets from function $\Lambda$.

Theorem 1.2. Assume (1.4) and (1.5) hold, then $\left\{\frac{\widetilde{S}_{n}}{b(n)}\right\}$ in $C([0,1], \mathbf{E})$ satisfies an MDP with speed $\left\{\frac{n}{b^{2}(n)}\right\}$ and rate function $\widetilde{\Lambda}$ defined by (1.2), that is, for every closed set $F$ and open set $G$ of $C([0,1], \mathbf{E})$,

$$
\limsup _{n \rightarrow \infty} \frac{n}{b^{2}(n)} \log P\left\{\frac{\widetilde{S}_{n}}{b(n)} \in F\right\} \leq-\inf _{f \in F} \widetilde{\Lambda}(f),
$$




$$
\liminf _{n \rightarrow \infty} \frac{n}{b^{2}(n)} \log P\left\{\frac{\widetilde{S}_{n}}{b(n)} \in G\right\} \geq-\inf _{g \in G} \widetilde{\Lambda}(g) .
$$

So does $\left\{\frac{S_{n}(\cdot)}{b(n)}\right\}$ in $D([0,1], \mathbf{E})$ with the same speed $\left\{\frac{n}{b^{2}(n)}\right\}$ and the same rate function $\widetilde{\Lambda}$.

Remark 1.2. From the proofs given in the next section, it can be seen that all lemmas, and thus Theorems 1.1 and 1.2 remain true if we substitute the underlying interval $[0,1]$ with interval $[0, T]$ for each $T>0$.

Remark 1.3. Theorem 1.2 has generalized the related results obtained by Borovkov and Mogulskii [12] and Deshayes and Picard [19].

If $\mathbf{E}$ is of type $p(1<p<2)$, then condition $E\left\|X_{1}\right\|^{p}<\infty$ implies $\frac{S_{n}}{n^{1 / p}} \stackrel{P}{\longrightarrow} 0$ (see Ledoux and Talagrand [27, pp. 190 and 259]). Therefore, an immediate corollary of Theorem 1.2 is following.

Corollary 1.1. Suppose $\mathbf{E}$ is of type $p, 1<p<2$, and $E \exp \left(\beta\left\|X_{1}\right\|\right)<\infty$ for some $\beta>0$. Then $\left\{\frac{\widetilde{S}_{n}}{n^{1 / p}}\right\}$ satisfies an $M D P$ in $C([0,1], \mathbf{E})$ with speed $\left\{\frac{n}{n^{2 / p}}\right\}$ and rate function $\widetilde{\Lambda}$ defined by (1.2).

It should be mentioned that the large deviation principles (LDPs) for $\left\{\widetilde{S}_{n} ; n \geq 1\right\}$ have been studied extensively, see Varadhan [32], Borovkov 10], Mogulskii [30], Deshayes and Picard 19, Borovkov and Mogulskii 12, Schuette 31, Dembo and Zajic [16, Hu 23] and the references therein. LDPs for sample paths of vectorvalued Lévy processes have also been proved by de Acosta 3]. Arcones 7] gave necessary and sufficient conditions for LDPs and MDPs for $\left\{S_{n}(\cdot) ; n \geq 1\right\}$ in the real-valued case.

The projective-limit method has been developed to successfully treat many problems. For a nice account of the theory, see, for example, Dembo and Zeitouni [18]. We do not see how to prove our result by the projective-limit method. A method of subsequences is devised to establish the MDP upper bound. The lower bound is proved by an interesting calculation which is rather explicit. Both upper and lower bounds are presented in Section 2. Our method is also different from that of Borovkov and Mogulskii [12. It would be interesting to see our problem worked out along the line of the projective-limit method; comparison of the two approaches should be instructive. On the other hand, it should also be interesting to look on the MDPs for stochastic processes in general, see Arcones [7] for corresponding results in real-valued case in this direction.

The Functional Laws of the Iterated Logarithm will be considered in Section 3. In Section 4, some remarks will be given.

\section{The PRoOfs of MAIN RESUlts}

We begin with several lemmas, which are important for proving the main results.

Lemma 2.1. Given $\varepsilon>0,0<\delta \leq 1$, under condition (1.5), for all sufficiently large $n$, we have

$$
P\left\{\max _{1 \leq k \leq[n \delta]+1}\left\|S_{k}\right\| \geq \varepsilon b(n)\right\} \leq 2 P\left\{\left\|S_{[n \delta]+1}\right\| \geq \frac{\varepsilon}{2} b(n)\right\},
$$




$$
P\left\{\max _{1 \leq k \leq n}\left\|S_{k}\right\| \geq \varepsilon b(n)\right\} \leq 2 P\left\{\left\|S_{n}\right\| \geq \frac{\varepsilon}{2} b(n)\right\} .
$$

Proof. We will prove only the first inequality, for the second can be treated similarly. By Ottaviani's inequality (see also Araujo and Gine, [6, pp. 110-111]), for every $A>0$ and every integer $M \geq 1$,

$$
P\left\{\max _{1 \leq k \leq M+1}\left\|S_{k}\right\| \geq A\right\} \leq \frac{P\left\{\left\|S_{M+1}\right\| \geq \frac{A}{2}\right\}}{1-\max _{1 \leq k \leq M} P\left\{\left\|S_{M+1-k}\right\| \geq \frac{A}{2}\right\}} .
$$

Take $M=[n \delta], A=\varepsilon b(n)$. By (1.5), we can steadily prove that for sufficiently large $n$,

$$
\max _{1 \leq k \leq[n \delta]} P\left\{\left\|S_{[n \delta]+1-k}\right\| \geq \frac{\varepsilon}{2} b(n)\right\} \leq \frac{1}{2},
$$

which implies the desired result. Lemma 2.1 is proved.

Lemma 2.2. Under condition (1.4), $\left\{\frac{\widetilde{S}_{n}(\cdot)}{b(n)}\right\}$ and $\left\{\frac{S_{n}(\cdot)}{b(n)}\right\}$ are exponentially equivalent in $D([0,1], \mathbf{E})$, that is, for each $\delta>0$,

$$
\limsup _{n \rightarrow \infty} \frac{n}{b^{2}(n)} \log P\left\{d_{\infty}\left(\frac{\widetilde{S}_{n}(\cdot)}{b(n)}, \frac{S_{n}(\cdot)}{b(n)}\right)>\delta\right\}=-\infty .
$$

Proof. Since $d_{\infty}\left(\widetilde{S}_{n}(\cdot), S_{n}(\cdot)\right) \leq \max _{1 \leq k \leq n}\left\|X_{k}\right\|$, by Chebyshev's inequality, for each $\delta>0$,

$$
\begin{aligned}
P\left\{d_{\infty}\left(\frac{\widetilde{S}_{n}(\cdot)}{b(n)}, \frac{S_{n}(\cdot)}{b(n)}\right)>\delta\right\} & \leq n P\left\{\left\|X_{1}\right\|>\delta b(n)\right\} \\
& \leq n \exp (-\beta \delta b(n)) E \exp \left(\beta\left\|X_{1}\right\|\right),
\end{aligned}
$$

where $\beta>0$ is as in (1.4), from which the exponential equivalence of $\left\{\frac{\widetilde{S}_{n}(\cdot)}{b(n)}\right\}$ and $\left\{\frac{S_{n}(\cdot)}{b(n)}\right\}$ follows. Lemma 2.2 is proved.

Following the proofs of Chen [13, Theorem 2]; [15, Theorem 1]), one can steadily prove the following version, Lemma 2.3, of the MDP for subsequences of i.i.d. sums.

Lemma 2.3. Let $\left\{Y_{j}\right\}$ be a sequence of i.i.d. random variables with values in $(\mathbf{E},\|\cdot\|)$, with common distribution $\mu \in W M_{0}^{2}$ and such that $E \exp \left(\alpha\left\|Y_{1}\right\|\right)<\infty$ for some $\alpha>0$. Let $\left\{n_{k}\right\}$ and $\left\{a_{k}\right\}$ be positive integers and positive numbers, respectively, such that as $k \rightarrow \infty$,

$$
n_{k} \rightarrow \infty, \quad \frac{a_{k}}{\sqrt{n_{k}}} \rightarrow \infty, \quad \frac{a_{k}}{n_{k}} \rightarrow 0
$$

and

$$
\frac{S_{n_{k}}}{a_{k}} \stackrel{P}{\longrightarrow} 0
$$

where $S_{n_{k}}=\sum_{j=1}^{n_{k}} Y_{j}, k \geq 1$.

Then $\left\{\frac{S_{n_{k}}}{a_{k}}\right\}$ satisfies an MDP with speed $\left\{\frac{n_{k}}{a_{k}^{2}}\right\}$ and the rate function $I(x)=$ $\Lambda(x)$, where $\Lambda$ is defined by (1.1). 
Lemma 2.4. For $0 \leq s<t \leq 1$ fixed, let $W_{n}(s, t)=S_{n}(t)-S_{n}(s)$. Assume (1.4) and (1.5) hold, then $\left\{\frac{W_{n}(s, t)}{b(n)}\right\}$ in $\mathbf{E}$ satisfies an $M D P$ with speed $\left\{\frac{n}{b^{2}(n)}\right\}$ and a rate function $I_{s, t}$, that is, for every closed set $F$ and open set $G$ of $\mathbf{E}$,

$$
\begin{aligned}
& \limsup _{k \rightarrow \infty} \frac{n}{b^{2}(n)} \log P\left\{\frac{W_{n}(s, t)}{b(n)} \in F\right\} \leq-\inf _{x \in F} I_{s, t}(x), \\
& \liminf _{k \rightarrow \infty} \frac{n}{b^{2}(n)} \log P\left\{\frac{W_{n}(s, t)}{b(n)} \in G\right\} \geq-\inf _{x \in G} I_{s, t}(x),
\end{aligned}
$$

where $I_{s, t}: \mathbf{E} \rightarrow[0, \infty]$ is defined as follows

$$
I_{s, t}(x)=(t-s) \Lambda\left(\frac{x}{t-s}\right)=\frac{1}{t-s} \Lambda(x), \quad x \in \mathbf{E} .
$$

Proof of Lemma 2.4. The function $I_{s, t}$ defined by (2.1) has compact level sets because $\Lambda$ does, see Goodman, Kuelbs and Zinn [22, Lemma 2.1(V)] and Chen [13. Theorem 2]; [15, Theorem 1].

Given $0 \leq s<t \leq 1$, define $n_{k}=[k t]-[k s]$ and $a_{k}=b(k), k \geq 1$. Then $W_{k}(s, t)$ has the same distribution as $S_{n_{k}}$ and hence $\frac{W_{k}(s, t)}{b(k)}$ has the same distribution as $\frac{S_{n_{k}}}{a_{k}}$. From (1.5) and Lemma 2.1 it follows that $\frac{S_{n_{k}}}{a_{k}} \stackrel{P}{\longrightarrow} 0$. Taking into account the fact that $\frac{k}{b^{2}(k)} \cdot \frac{a_{k}^{2}}{n_{k}} \rightarrow \frac{1}{t-s}$ as $k \rightarrow \infty$, Lemma 2.4 follows from Lemma 2.3. Lemma 2.4 is proved.

Proof of Theorem 1.1. Given a partition, $\pi$, of $[0,1], \pi: 0=t_{0}<t_{1}<\cdots<t_{N}=1$, denote $W_{n}^{\pi}=\left(S_{n}\left(t_{1}\right), S_{n}\left(t_{2}\right)-S_{n}\left(t_{1}\right), \cdots, S_{n}\left(t_{N}\right)-S_{n}\left(t_{N-1}\right)\right)$. Then by Lemma 2.4, Lynch and Sethuraman [28, Corollary 2.9] (see also Dembo and Zeitouni [18, Ex. 4.2.7]), $\left\{\frac{W_{n}^{\pi}}{b(n)}\right\}$ satisfies an MDP in $\mathbf{E}^{N}$ with speed $\left\{\frac{n}{b^{2}(n)}\right\}$ and rate function $I^{\pi}$ given by

$$
I^{\pi}(z)=\sum_{i=1}^{N}\left(t_{i}-t_{i-1}\right) \Lambda\left(\frac{z_{i}}{t_{i}-t_{i-1}}\right)
$$

for $z=\left(z_{1}, \cdots, z_{N}\right) \in \mathbf{E}^{N}$.

Applying the contraction principle (see Dembo and Zeitouni [18, Theorem 4.2.1]) to the continuous one-to-one map $\left(z_{1}, \cdots, z_{N}\right) \rightarrow\left(z_{1}, z_{1}+z_{2}, \cdots, \sum_{i=1}^{N} z_{i}\right)$ on $\mathbf{E}^{N}$, from the MDP for the sequence $\left\{\frac{W_{n}^{\pi}}{b(n)}\right\}$ it follows that $\left\{\frac{1}{b(n)}\left(S_{n}\left(t_{1}\right), \cdots, S_{n}\left(t_{N}\right)\right)\right\}$ satisfies an MDP in $\mathbf{E}^{N}$ with speed $\left\{\frac{n}{b^{2}(n)}\right\}$ and rate function defined by the equality (1.8). Lemma 2.2 implies that the sequences $\left\{\frac{1}{b(n)}\left(S_{n}\left(t_{1}\right), \cdots, S_{n}\left(t_{N}\right)\right)\right\}$ and $\left\{\frac{1}{b(n)}\left(\widetilde{S}_{n}\left(t_{1}\right), \cdots, \widetilde{S}_{n}\left(t_{N}\right)\right)\right\}$ in $\mathbf{E}^{N}$ are exponentially equivalent. Hence, by Dembo and Zeitouni [18 Theorem 4.2.13], $\left\{\frac{1}{b(n)}\left(\widetilde{S}_{n}\left(t_{1}\right), \cdots, \widetilde{S}_{n}\left(t_{N}\right)\right)\right\}$ in $\mathbf{E}^{N}$ satisfies the same MDP as that for $\left\{\frac{1}{b(n)}\left(S_{n}\left(t_{1}\right), \cdots, S_{n}\left(t_{N}\right)\right)\right\}$. Theorem 1.1 is proved.

Proof of Theorem 1.2. The compactness of level sets of $\widetilde{\Lambda}$ will be proved in Appendix B. 
Upper bound. We first show that for every $f \in C([0,1], \mathbf{E})$, and every $\epsilon>0$, there exists a ball of $f, B(f, \rho)=\left\{g ;\|f-g\|_{\infty}<\rho\right\}$ for some $\rho=\rho(f, \epsilon)>0$, such that

$$
\limsup _{n \rightarrow \infty} \frac{n}{b^{2}(n)} \log P\left\{\frac{\widetilde{S}_{n}(\cdot)}{b(n)} \in B(f, \rho)\right\} \leq-\widetilde{\Lambda}(f)+\epsilon .
$$

To this end, given $f \in C([0,1], \mathbf{E})$ and $\varepsilon>0$, we first consider the case where $\widetilde{\Lambda}(f)<\infty$.

For every partition, $\pi$, of $[0,1], \pi: 0=t_{0}<t_{1}<\cdots<t_{N}=1$, by Theorem 1.1,

$$
\begin{gathered}
\limsup _{n \rightarrow \infty} \frac{n}{b^{2}(n)} \log P\left\{\frac{\widetilde{S}_{n}(\cdot)}{b(n)} \in B(f, \rho)\right\} \\
\leq \limsup _{n \rightarrow \infty} \frac{n}{b^{2}(n)} \log P\left\{\frac{\widetilde{S}_{n}\left(t_{i}\right)}{b(n)} \in \overline{B_{\rho}\left(f\left(t_{i}\right)\right)}, i=1, \cdots, N\right\} \\
\leq-\inf \left\{\widetilde{I}^{\pi}(z) ; z=\left(z_{1}, \cdots, z_{N}\right), z_{i} \in \overline{B_{\rho}\left(f\left(t_{i}\right)\right)}, i=1, \cdots, N\right\}
\end{gathered}
$$

where $B_{\rho}(x)=\{y \in \mathbf{E} ;\|x-y\|<\rho\}$ for $x \in \mathbf{E}$, and $\widetilde{I}^{\pi}(\cdot)$ is as in (1.8). We can choose partition $\pi$ such that

$$
\widetilde{I}^{\pi}\left(\left(f\left(t_{1}\right), \cdots, f\left(t_{N}\right)\right)\right) \geq \widetilde{\Lambda}(f)-\epsilon / 2 .
$$

By the lower semicontinuity of $\widetilde{I}^{\pi}$, we can choose $\rho=\rho(f, \epsilon)>0$ small enough such that

$$
\begin{gathered}
\inf \left\{\widetilde{I}^{\pi}(z) ; z=\left(z_{1}, \cdots, z_{N}\right), z_{i} \in \overline{B_{\rho}\left(f\left(t_{i}\right)\right)}, 1 \leq i \leq N\right\} \\
\geq \inf \left\{\widetilde{I}^{\pi}(z) ; z=\left(z_{1}, \cdots, z_{N}\right), z_{i} \in B_{2 \rho}\left(f\left(t_{i}\right)\right), 1 \leq i \leq N\right\} \\
\geq \widetilde{I}^{\pi}\left(\left(f\left(t_{1}\right), \cdots, f\left(t_{N}\right)\right)\right)-\epsilon / 2 .
\end{gathered}
$$

So (2.2) follows from (2.3)-(2.5). If $\widetilde{\Lambda}(f)=+\infty$, then we can similarly prove that (2.2) is still true.

Keeping (2.2) in mind, by a well-known standard argument we know that the upper bound holds for compact sets. Therefore, in order to complete the upper bound, it suffices to prove that $\left\{\frac{\widetilde{S}_{n}(\cdot)}{b(n)}\right\}$ is exponentially tight, that is, for every $L>0$, there exists a compact set $K_{L} \subset C([0,1], \mathbf{E})$, such that

$$
\limsup _{n \rightarrow \infty} \frac{n}{b^{2}(n)} \log P\left\{\frac{\widetilde{S}_{n}(\cdot)}{b(n)} \in K_{L}^{c}\right\} \leq-L .
$$

We will adapt Dembo and Zajic [16]'s argument to prove (2.6). However, a much more delicate estimate is needed when we prove (2.7) below.

By Theorem A in the Appendix A it suffices to prove

(i) For each rational $t \in[0,1],\left\{\frac{\widetilde{S}_{n}(t)}{b(n)}\right\}$ is exponentially tight, that is, for each $\alpha>0$, there exists a compact set $K_{\alpha} \subset \mathbf{E}$ such that

$$
\limsup _{n \rightarrow \infty} \frac{n}{b^{2}(n)} \log P\left\{\frac{\widetilde{S}_{n}(t)}{b(n)} \in K_{\alpha}^{c}\right\} \leq-\alpha .
$$


(ii) For each $\rho>0$,

$$
\lim _{\delta \rightarrow 0} \limsup _{n \rightarrow \infty} \frac{n}{b^{2}(n)} \log P\left\{\frac{1}{b(n)} \sup _{|t-s|<\delta}\left\|\widetilde{S}_{n}(t)-\widetilde{S}_{n}(s)\right\| \geq \rho\right\}=-\infty .
$$

To this end. (i) follows from Lemma 2.4 and Lynch and Sethuraman [28, Lemma $2.6]$.

To prove (2.7). Noting Lemma 2.2, it is enough to prove

$$
\lim _{\delta \rightarrow 0} \limsup _{n \rightarrow \infty} \frac{n}{b^{2}(n)} \log P\left\{\frac{1}{b(n)} \sup _{|t-s|<\delta}\left\|S_{n}(t)-S_{n}(s)\right\| \geq \rho\right\}=-\infty
$$

for each $\rho>0$.

In fact, fix $\rho>0$, for $n \geq 1$ and $\delta>0$,

$$
\sup _{|t-s|<\delta}\left\|S_{n}(t)-S_{n}(s)\right\| \leq \max _{0 \leq k \leq n, 1 \leq m \leq[n \delta]+1}\left\|\sum_{j=k+1}^{k+m} X_{j}\right\| .
$$

For $0<\delta \leq 1$, let $l=l(\delta) \geq 2$ be the unique integer satisfying $1 / l<\delta \leq 1 /(l-1)$. Then $n<([n \delta]+1) l$ for sufficiently large $n$. For such a large $n$, suppose

$$
\left\|S_{k+m}-S_{k}\right\|=\left\|\sum_{j=k+1}^{k+m} X_{j}\right\| \geq b(n) \rho
$$

for some $k, 0 \leq k \leq n$, and some $m, 1 \leq m \leq[n \delta]+1$. Then there exists a unique integer $p, 0 \leq p \leq l-1$, such that

$$
([n \delta]+1) p \leq k<([n \delta]+1)(p+1) .
$$

Hence, there are two possibilities for $k+m$. One possibility is that

$$
([n \delta]+1) p \leq k+m<([n \delta]+1)(p+1),
$$

in which case either $\left\|S_{k+m}-S_{([n \delta]+1) p}\right\| \geq \frac{1}{3} b(n) \rho$, or $\left\|S_{k}-S_{([n \delta]+1) p}\right\| \geq \frac{1}{3} b(n) \rho$. The second possibility is that

$$
([n \delta]+1)(p+1) \leq k+m<([n \delta]+1)(p+2),
$$

in which case either $\left\|S_{k+m}-S_{([n \delta]+1)(p+1)}\right\| \geq \frac{1}{3} b(n) \rho,\left\|S_{([n \delta]+1)(p+1)}-S_{([n \delta]+1) p}\right\| \geq$ $\frac{1}{3} b(n) \rho$, or $\left\|S_{k}-S_{([n \delta]+1) p}\right\| \geq \frac{1}{3} b(n) \rho$. In conclusion, we see that

$$
\begin{gathered}
\left\{\max _{0 \leq k \leq n, 1 \leq m \leq[n \delta]+1}\left\|\sum_{j=k+1}^{k+m} X_{j}\right\| \geq b(n) \rho\right\} \\
\subset \sum_{p=0}^{l}\left\{\max _{1 \leq m \leq[n \delta]+1}\left\|\sum_{j=p([n \delta]+1)+1}^{p([n \delta]+1)+m} X_{j}\right\| \geq \frac{\rho}{3} b(n)\right\} .
\end{gathered}
$$

Noting

$$
\begin{array}{r}
P\left\{\max _{1 \leq m \leq[n \delta]+1}\left\|\sum_{j=p([n \delta]+1)+1}^{p([n \delta]+1)+m} X_{j}\right\| \geq \frac{\rho}{3} b(n)\right\} \\
=P\left\{\max _{1 \leq m \leq[n \delta]+1}\left\|\sum_{j=1}^{m} X_{j}\right\| \geq \frac{\rho}{3} b(n)\right\},
\end{array}
$$


by (2.9), (2.10) and Lemma 2.1,

$$
\begin{gathered}
P\left\{\frac{1}{b(n)} \sup _{|t-s|<\delta}\left\|S_{n}(t)-S_{n}(s)\right\| \geq \rho\right\} \\
\leq \sum_{p=0}^{l} P\left\{\frac{1}{b(n)} \max _{1 \leq m \leq[n \delta]+1}\left\|\sum_{j=1}^{m} X_{j}\right\| \geq \frac{\rho}{3}\right\} \\
\leq 2(l+1) P\left\{\frac{1}{b(n)}\left\|\sum_{j=1}^{\| n \delta]+1} X_{j}\right\| \geq \frac{\rho}{6}\right\} \\
\leq 2(l+1) P\left\{\frac{\left\|S_{[n \delta]}\right\|}{b(n)} \geq \frac{\rho}{12}\right\}+2(l+1) P\left\{\frac{\left\|X_{1}\right\|}{b(n)} \geq \frac{\rho}{12}\right\} .
\end{gathered}
$$

By (2.11), (1.4), Lemma 2.4 and Chebyshev's inequality, for all $0<\delta \leq 1$,

$$
\begin{gathered}
\limsup _{n \rightarrow \infty} \frac{n}{b^{2}(n)} \log P\left\{\frac{1}{b(n)} \sup _{|t-s|<\delta}\left\|S_{n}(t)-S_{n}(s)\right\| \geq \rho\right\} \\
\leq \max \left\{\limsup _{n \rightarrow \infty} \frac{n}{b^{2}(n)} \log P\left\{\frac{\left\|S_{[n \delta]}\right\|}{b(n)} \geq \frac{\rho}{12}\right\},\right. \\
\left.\quad \limsup _{n \rightarrow \infty} \frac{n}{b^{2}(n)} \log P\left\{\left\|X_{1}\right\| \geq \frac{\rho}{12} b(n)\right\}\right\} \\
\leq-\frac{1}{\delta} \inf \left\{\frac{1}{2}\|x\|_{H}^{2} ; x \in H,\|x\| \geq \frac{\rho}{12}\right\} .
\end{gathered}
$$

Note that for $x \in H$,

$$
\|x\| \leq \sigma\|x\|_{H}
$$

where

$$
\sigma=\sup _{\|g\| \leq 1, g \in \mathbf{E}^{*}}\left(\int_{\Omega} g^{2}\left(X_{1}\right) d P\right)^{1 / 2}<\infty .
$$

(See Goodman, Kuebls and Zinn [22].) Taking limit $\delta \rightarrow 0$ in (2.12) implies (2.8). The upper bound is established.

Lower bound. To prove the lower bound, it suffices to prove that for each piecewise linear function $f \in C([0,1], \mathbf{E})$, and each $\rho>0$,

$$
\liminf _{n \rightarrow \infty} \frac{n}{b^{2}(n)} \log P\left\{\frac{\widetilde{S}_{n}(\cdot)}{b(n)} \in B(f, \rho)\right\} \geq-\widetilde{\Lambda}(f) .
$$

(See also Schuette [31], Hu [23], etc.)

This can be easily reduced to proving for the case of a linear function $f: t \rightarrow$ $t x, t \in[0,1], x \in \mathbf{E}$ (see also Borovkov and Mogulskii [12]). So, we now focus on the case $f(t)=t x, t \in[0,1]$, where $x \in \mathbf{E}$ is arbitrarily fixed.

For $\theta>0, x \in \mathbf{E}$, let $N_{\theta}(x)=\{g \in C([0,1], \mathbf{E}) ;\|g(1)-x\|<\theta\}$. Note that for $\rho>0, \theta>0$,

$$
\overline{N_{\theta}(x)} \cap B(f, \rho)=\overline{N_{\theta}(x)} \backslash\left\{\overline{N_{\theta}(x)} \backslash B(f, \rho)\right\} \subset B(f, \rho) .
$$


Since the MDP for the partial sums $\left\{\frac{\widetilde{S}_{n}(1)}{b(n)}\right\}$ yields

$$
\begin{gathered}
\liminf _{n \rightarrow \infty} \frac{n}{b^{2}(n)} \log P\left\{\frac{\widetilde{S}_{n}(\cdot)}{b(n)} \in \overline{N_{\theta}(x)}\right\} \\
\geq \liminf _{n \rightarrow \infty} \frac{n}{b^{2}(n)} \log P\left\{\left\|\frac{\widetilde{S}_{n}(1)}{b(n)}-x\right\|<\theta\right\} \\
\geq-\Lambda(x)=-\widetilde{\Lambda}(f),
\end{gathered}
$$

in order to prove $(2.14)$, it suffices to prove

$$
\limsup _{n \rightarrow \infty} \frac{n}{b^{2}(n)} \log P\left\{\frac{\widetilde{S}_{n}(\cdot)}{b(n)} \in \overline{N_{\theta}(x)} \backslash B(f, \rho)\right\}<-\Lambda(x)
$$

for a certain $\theta>0$.

A little thought reveals that all sufficiently small $\theta>0$ will do. Since $\overline{N_{\theta}(x)} \backslash$ $B(f, \rho)$ is a closed set, we apply the upper bound result and estimate

$$
\begin{aligned}
& -(\text { L. H. S. of }(2.15)) \geq \inf \{\widetilde{\Lambda}(g) ;\|g-f\| \geq \rho,\|g(1)-x\| \leq \theta\} \\
& \geq \inf \left\{\widetilde{\Lambda}(g) ; \sup _{0 \leq t \leq 1}\|g(t)-(f(t)+t z)\| \geq \rho-\theta, z=g(1)-x,\|z\| \leq \theta\right\} \\
& =\inf _{\|z\| \leq \theta} \inf \{\widetilde{\Lambda}(g) ;\|g(t)-t(x+z)\| \geq \rho-\theta \text { for some } 0 \leq t \leq 1, g(1)=x+z\} \\
& \geq \inf _{\|z\| \leq \theta} \inf _{0 \leq t \leq 1} \inf \{\widetilde{\Lambda}(g) ; y=g(t)-t(x+z),\|y\| \geq \rho-\theta, g(1)=x+z\} \\
& \geq \inf _{\|z\| \leq \theta} \inf _{0 \leq t \leq 1} \inf _{\|y\| \geq \rho-\theta}\left\{t \Lambda\left((x+z)+\frac{y}{t}\right)+(1-t) \Lambda\left((x+z)-\frac{y}{1-t}\right)\right\} \\
& \geq \inf _{\|z\| \leq \theta} \inf _{\|y\|_{H} \geq \frac{\rho-\theta}{\sigma}} \inf _{0 \leq t \leq 1} \frac{1}{2}\left\{t\left\|x+z+\frac{y}{t}\right\|_{H}^{2}+(1-t)\left\|x+z-\frac{y}{1-t}\right\|_{H}^{2}\right\} \\
& \geq \inf _{\|z\| \leq \theta} \inf _{\|y\|_{H} \geq \frac{\rho-\theta}{\sigma}} \inf _{0 \leq t \leq 1} \frac{1}{2}\left\{\|x+z\|_{H}^{2}+\left(\frac{1}{t}+\frac{1}{1-t}\right)\|y\|_{H}^{2}\right\} \\
& \geq \inf _{\|z\| \leq \theta}\left\{\frac{1}{2}\|x+z\|_{H}^{2}+2\left(\frac{\rho-\theta}{\sigma}\right)^{2}\right\} \\
& =\inf _{\|z\| \leq \theta}\left\{\Lambda(x+z)+2\left(\frac{\rho-\theta}{\sigma}\right)^{2}\right\}
\end{aligned}
$$

whose limit as $\theta \rightarrow 0$ is greater than or equal to $\Lambda(x)+2\left(\frac{\rho}{\sigma}\right)^{2}$ by the lower semicontinuity of the rate function $\Lambda$, where $\sigma$ is as in (2.13).

This concludes that (2.15) indeed holds for sufficiently small $\theta>0$. The proof of the lower bound is completed.

So far, we have proved that $\left\{\frac{\widetilde{S}_{n}(\cdot)}{b(n)}\right\}$ in $C([0,1], \mathbf{E})$ satisfies an MDP with speed $\left\{\frac{n}{b^{2}(n)}\right\}$ and the rate function $\widetilde{\Lambda}$ defined by (1.2). It is easy to check that $(C([0,1], \mathbf{E})$, $\left.\|\cdot\|_{\infty}\right)$ is a closed subset of $\left(D([0,1], \mathbf{E}), d_{\infty}\right)$, and therefore $\left\{\frac{\widetilde{S}_{n}(\cdot)}{b(n)}\right\}$ in $(D([0,1], \mathbf{E})$, $\left.d_{\infty}\right)$ also satisfies the MDP with speed $\left\{\frac{n}{b^{2}(n)}\right\}$ and the rate function $\widetilde{\Lambda}$ of $(1.2)$ (see Dembo and Zeitouni [18, Lemma 4.1.5 (a)]. The exponential equivalence of 
$\left\{\frac{\widetilde{S}_{n}(\cdot)}{b(n)}\right\}$ and $\left\{\frac{S_{n}(\cdot)}{b(n)}\right\}$ in $\left(D([0,1], \mathbf{E}), d_{\infty}\right)$, established in Lemma 2.2, implies now that $\left\{\frac{S_{n}(\cdot)}{b(n)}\right\}$ in $D([0,1], \mathbf{E})$ satisfies the $\operatorname{MDP}$ with speed $\left\{\frac{n}{b^{2}(n)}\right\}$ and the rate function $\widetilde{\Lambda}$ of (1.2) (see Dembo and Zeitouni [18, Theorem 4.2.13]). Theorem 1.2 is proved.

\section{FunCtional LiLs}

Throughout this section, let $\beta(n)=\sqrt{2 n \log \log n}, n \geq 3$. Based on Theorem 1.2 and Remark 1.2, we can conclude the Functional Laws of the Iterated Logarithm for the piecewise constant and piecewise linear functions, $\left\{S_{n}(\cdot) ; n \geq 1\right\}$ and $\left\{\widetilde{S}_{n}(\cdot) ; n \geq 1\right\}$, respectively.

Theorem 3.1. Suppose that (1.4) and the following condition

$$
\frac{S_{n}}{\beta(n)} \stackrel{P}{\longrightarrow} 0
$$

hold. Then with probability 1 , the following sequence

$$
\left\{\xi_{n}(\cdot)=\frac{S_{n}(\cdot)}{\beta(n)}\right\}_{n \geq 1}
$$

is relatively compact in $D([0,1], \mathbf{E})$, and the set of its limit points, $L(\omega)$, is precisely the compact set

$$
\mathcal{K}=\{f \in D([0,1], \mathbf{E}) ; 2 \widetilde{\Lambda}(f) \leq 1\}
$$

The same result holds for $\left\{\frac{\widetilde{S}_{n}(\cdot)}{\beta(n)}\right\}_{n \geq 1}$.

Proof. It can be proved by the standard arguments, see, for example, the proof of Deuschel and Stroock [20, Theorem 1.4.1] or Dembo and Zajic [17, Corollary 1].

\section{CONCLUding REMARKS}

We have viewed the trajectory problem as consisting of two major issues. First, what is the MDP for partial sums? For this we found good results in the literature, see, for instance, Chen [13] 15], Ledoux [26]. Second, how to pass a result from the partial sum to the whole trajectory, now that the result holds for partial sums? The latter issue is treated carefully in Section 2.

We have traced all the proofs and seen that, once the MDP for partial sums is assumed, the original assumption (1.4) is rarely quoted in settling the second issue. This, among other things, suggests that any partial sum result may well remain true for the corresponding trajectory process. Let us illustrate use of such an idea by extending a partial sum result to the trajectory setting, the results in Proposition 4.1 and Proposition 4.2 below. In the proof, we will list all occasions of quoting the original assumption, (1.4). It should be pointed out that (1.5) is a necessary condition for (1.6) to hold.

Proposition 4.1. Let $b(n)=n^{p}(1 / 2<p<1)$. Suppose that (1.5) and

$$
E \exp \left(\beta\left\|X_{1}\right\|^{\alpha}\right)<\infty \text { for some } 2-1 / p<\alpha<1 \text { and some } \beta=\beta(\alpha)>0
$$

hold. Then Theorem 1.1 and Theorem 1.2 remain true. 
Proof. By Chen [13, Theorem 2]; [15, Theorem 1] or Jiang [24], $\left\{\frac{S_{n}}{b(n)}\right\}$ satisfies an MDP with speed $\left\{\frac{n}{b^{2}(n)}\right\}$ and rate function $\Lambda$ as in (1.1).

Again by tracing the proofs of Chen [13, Theorem 2]; 15, Theorem 1], we can show that Lemma 2.3 is still true under the new assumption instead of (1.4).

Excluding Lemma 2.3, use was made of (1.4) only in the proofs of Lemma 2.2 and (2.12) via the following estimate

$$
P\left\{\left\|X_{1}\right\|>b(n) \delta\right\} \leq \exp (-\beta \delta b(n)) E \exp \left(\beta\left\|X_{1}\right\|\right)
$$

for $\delta>0$, where $\beta$ is as in (1.4). The inequality above ensures the desired estimate

$$
\limsup _{n \rightarrow \infty} \frac{n}{b^{2}(n)} \log \left(n P\left\{\left\|X_{1}\right\|>b(n) \delta\right\}\right)=-\infty
$$

for each $\delta>0$. Therefore, all that remains is to show that (4.1) is valid under the new assumption, weaker than (1.4). Indeed, for each $\delta>0$, by Chebyshev's inequality,

$$
n P\left\{\left\|X_{1}\right\|>\delta b(n)\right\} \leq n \exp \left(-\beta \delta^{\alpha} b^{\alpha}(n)\right) E \exp \left(\beta\left\|X_{1}\right\|^{\alpha}\right),
$$

which implies (4.1). Proof is completed.

Proposition 4.2. Let $b(n)=\sqrt{2 n \log \log n}, n \geq 3$. Suppose that (1.5) and

$$
E \exp \left(\beta\left\|X_{1}\right\|^{\alpha}\right)<\infty \text { for some } 0<\alpha<1 \text { and some } \beta=\beta(\alpha)>0
$$

hold. Then Theorem 1.1, Theorem 1.2 and Theorem 3.1 hold.

Proof. Its proof is similar to that of Proposition 4.1.

Remark 4.1. Proposition 4.2 has improved Theorem 3.1 by weakening the exponential integrability assumption, (1.4).

\section{Appendix A}

Let $(\mathcal{X}, d)$ be a Polish space and $\mathcal{Y}$ denote the Polish space of continuous functions from $[0,1]$ to $\mathcal{X}$ equipped with the metric $d_{\infty}(f, g)=\sup _{0 \leq t \leq 1} d(f(t), g(t))$. Let $\{a(n)\}$ be a positive sequence satisfying $a(n) \rightarrow 0$ as $n \rightarrow \infty$.

A sequence of probability measures $\left\{\mu_{n} ; n \geq 1\right\}$ on $\mathcal{Y}$ is said to be exponentially tight with speed $\{a(n)\}$ if for every $L>0$, there exists a compact set $K_{L} \subset \mathcal{Y}$ such that

$$
\limsup _{n \rightarrow \infty} a(n) \log \mu_{n}\left\{K_{L}^{c}\right\} \leq-L,
$$

where $K_{L}^{c}$ means the complement of $K_{L}$.

Theorem A. A sequence of probability measures $\left\{\mu_{n} ; n \geq 1\right\}$ on $\mathcal{Y}$ is exponentially tight with speed $\{a(n)\}$ if;

(i) For each rational $t \in[0,1]$, the sequence $\left\{\mu_{n}(t) ; n \geq 1\right\}$ of laws induced by the projection $f(\cdot) \rightarrow f(t): \mathcal{Y} \rightarrow \mathcal{X}$ is exponentially tight in $(\mathcal{X}, d)$, that is, for each $\alpha>0$, there exists a compact set $L_{\alpha} \subset \mathcal{X}$ such that

$$
\limsup _{n \rightarrow \infty} a(n) \log \mu_{n}\left\{f(t) \in L_{\alpha}^{c}\right\} \leq-\alpha .
$$

(ii) For all $\rho>0$,

$$
\lim _{\delta \rightarrow 0} \limsup _{n \rightarrow \infty} a(n) \log \mu_{n}\left\{\left\{f ; \omega_{f}(\delta) \geq \rho\right\}\right\}=-\infty
$$


where for each $f \in \mathcal{Y}$ and all $\delta>0$,

$$
\omega_{f}(\delta)=\sup _{|t-s|<\delta} d(f(t), f(s))
$$

denotes the modulus of continuity of $f$.

Proof. This is essentially the Lemma A.2 of Dembo and Zajic [16].

\section{Appendix B}

Let $\widetilde{\Lambda}$ be defined by (1.2). The purpose of this section is to show that $\widetilde{\Lambda}$, defined on $\left(D([0,1], \mathbf{E}), d_{\infty}(\cdot, \cdot)\right)$, has compact level sets. Since $\left(C([0,1], \mathbf{E}),\|\|_{\infty}\right)$ is a closed subspace of $\left(D([0,1], \mathbf{E}), d_{\infty}(\cdot, \cdot)\right)$, it suffices for us to show that $\widetilde{\Lambda}$ restricted to $\left(C([0,1], \mathbf{E}),\|\cdot\|_{\infty}\right)$ has compact level sets. Throughout this section, we denote by $\lambda$ the Lebesque measure on $[0,1]$. Note that $\Lambda$ has compact level sets under the integrability condition (1.4).

Lemma B.1. Let $K_{a}=\{\varphi \in C([0,1] ; \mathbf{E}) ; \widetilde{\Lambda}(\varphi) \leq a\}$ for $a>0$, and let $\dot{K}_{a}=\{g \in$ $L^{1}([0,1], \mathbf{E}) ; \varphi(t)=\int_{0}^{t} g(s) d s$ for $\left.t \in[0,1], \varphi \in K_{a}\right\}$. Then $\dot{K}_{a}$ is $\|\cdot\|_{H}$-uniformly integrable, that is to say

$$
\lim _{k \rightarrow \infty} \sup _{g \in \dot{K}_{a}} \int_{\left\{\|g\|_{H} \geq k\right\}}\|g\|_{H} d \lambda=0 .
$$

Proof. Given $g \in \dot{K}_{a}$, for each $k>0$, it follows from the Cauchy-Schwartz inequality that

$$
\int_{\left\{\|g\|_{H} \geq k\right\}}\|g\|_{H} d \lambda \leq \frac{2}{k} \widetilde{\Lambda}(\varphi) \leq \frac{2}{k} a,
$$

where $\varphi(t)=\int_{0}^{t} g(s) d s$ for $t \in[0,1]$. (B.1) follows from (B.2). The lemma is proved.

Lemma B.2 (Lower semicontinuity of $\widetilde{\Lambda}$ ). If $\left\|\varphi_{n}-\varphi\right\|_{\infty} \rightarrow 0$ as $n \rightarrow \infty$, then $\liminf _{n \rightarrow \infty} \widetilde{\Lambda}\left(\varphi_{n}\right) \geq \widetilde{\Lambda}(\varphi)$.

Proof. It suffices to consider $\liminf _{n \rightarrow \infty} \widetilde{\Lambda}\left(\varphi_{n}\right)=b<\infty$. By passing to a subsequence, we may and will assume that $\lim _{n \rightarrow \infty} \widetilde{\Lambda}\left(\varphi_{n}\right)=b$, and $\widetilde{\Lambda}\left(\varphi_{n}\right) \leq b+1$ for all $n$. Let $g_{n} \in L^{1}([0,1], \mathbf{E})$ such that $g_{n}(t) \in H$ and $\varphi_{n}(t)=\int_{0}^{t} g_{n}(s) d s$ for $t \in[0,1]$.

We shall show that $\varphi$ is $\|\cdot\|_{H}$-absolutely continuous: that is, for every $\varepsilon>0$, there exists $\delta=\delta(\varepsilon)>0$ such that $n \in \mathbf{N}, 0 \leq s_{1}<t_{1} \leq s_{2}<t_{2} \leq \cdots \leq s_{n}<t_{n} \leq$ $1, \sum\left(t_{i}-s_{i}\right)<\delta$ imply

$$
\sum_{i=1}^{n}\left\|\varphi\left(t_{i}\right)-\varphi\left(s_{i}\right)\right\|_{H}<\varepsilon
$$

To prove (B.3). Given $\varepsilon>0$, by Lemma B.1, there exists $\delta=\delta(\varepsilon)>0$ such

that for all $n, \int_{A}\left\|g_{n}\right\|_{H} d \lambda<\varepsilon$ whenever $\lambda(A)<\delta$. In particular, if $s_{i}<t_{i}$ and $\sum\left|t_{i}-s_{i}\right|<\delta$, then

$$
\sum\left\|\varphi_{n}\left(t_{i}\right)-\varphi_{n}\left(s_{i}\right)\right\|_{H}<\varepsilon
$$


Taking account of (B.4), the lower semicontinuity of $\Lambda$ and $\left\|\varphi_{n}-\varphi\right\|_{\infty} \rightarrow 0$, we can obtain

(B.5) $\varepsilon \geq \liminf _{n \rightarrow \infty} \sum\left(2 \Lambda\left(\varphi_{n}\left(t_{i}\right)-\varphi_{n}\left(s_{i}\right)\right)\right)^{1 / 2} \geq \sum\left(2 \Lambda\left(\varphi\left(t_{i}\right)-\varphi\left(s_{i}\right)\right)\right)^{1 / 2}$.

Note that $\varphi(0)=\lim _{n \rightarrow \infty} \varphi_{n}(0)=0 \in H$. (B.5), together with the definition of $\Lambda$, implies that $\varphi\left(s_{i}\right), \varphi\left(t_{i}\right) \in H$. In return, we have $\varphi(t) \in H$ for all $t \in[0,1]$ and

$$
\sum\left\|\varphi\left(t_{i}\right)-\varphi\left(s_{i}\right)\right\|_{H} \leq \varepsilon
$$

which means that $\varphi$ is $\|\cdot\|_{H}$-absolutely continuous. Note that (B.6) yields also that $\int_{0}^{1}\|\varphi(s)\|_{H} d s<\infty$.

We next show that there exists $g \in L^{1}([0,1], \mathbf{E})$ such that $g(t) \in H$ and $\varphi(t)=$ $\int_{0}^{t} g(s) d s$ for $t \in[0,1]$. To this end, define on $([0,1], \mathcal{B}, \lambda)$, where $\mathcal{B}$ is the Borel $\sigma$-algebra of $[0,1]$, the $H$-valued martingale $\left(h_{n}, \mathcal{F}_{n}\right)$, where

$$
h_{n}=\sum_{j=1}^{2^{n}} 2^{n}\left[\varphi\left(\frac{j}{2^{n}}\right)-\varphi\left(\frac{j-1}{2^{n}}\right)\right] \mathbf{1}_{\left[(j-1) / 2^{n}, j / 2^{n}\right)}
$$

and $\mathcal{F}_{n}=\sigma\left(\left[\frac{j-1}{2^{n}}, \frac{j}{2^{n}}\right) ; 1 \leq j \leq 2^{n}\right)$.

Because $\varphi$ is $\|\cdot\|_{H}$-absolutely continuous, it is of $\|\cdot\|_{H}$-bounded variation; that is, there exists a positive constant $M<\infty$, such that if $n \in \mathbf{N}$ and $0 \leq t_{0}<t_{1}<$ $\cdots<t_{n} \leq 1$, then

$$
\sum_{i=1}^{n}\left\|\varphi\left(t_{i}\right)-\varphi\left(t_{i-1}\right)\right\|_{H} \leq M
$$

Since for $n \in \mathbf{N}$,

$$
E\left\|h_{n}\right\|_{H}=\sum_{j=1}^{2^{n}}\left\|\varphi\left(\frac{j}{2^{n}}\right)-\varphi\left(\frac{j-1}{2^{n}}\right)\right\|_{H},
$$

it follows from (B.7) that $\sup _{n} E\left\|h_{n}\right\|_{H}<\infty$. By the well-known martingale convergence theorem, there exists $g \in L^{1}([0,1], \mathbf{E})$ satisfying $g(t) \in H$ for $t \in[0,1]$ and $\int_{0}^{1}\|g(s)\|_{H} d s<\infty$ such that $\lim _{n \rightarrow \infty}\left\|h_{n}-g\right\|_{H}=0$ a.e. Next, we shall show that $\varphi(t)=\int_{0}^{t} g(s) d s$ for $t \in[0,1]$. First of all, we shall show that

$$
\lim _{n \rightarrow \infty} E\left\|h_{n}-g\right\|_{H}=0 .
$$

To prove (B.8), it is enough to show $\left\{h_{n}\right\}$ is $\|\cdot\|_{H^{-}}$uniformly integrable, that is

$$
\lim _{\rho \rightarrow \infty} \sup _{n} \int_{\left\{\left\|h_{n}\right\|_{H} \geq \rho\right\}}\left\|h_{n}\right\|_{H} d \lambda=0 .
$$

To show this, let $\triangle_{j}=\varphi\left(\frac{j}{2^{n}}\right)-\varphi\left(\frac{j-1}{2^{n}}\right), j=1, \cdots, 2^{n}$, then

$$
C \triangleq \sup _{n} \sum_{j=1}^{2^{n}}\left\|\triangle_{j}\right\|_{H}<\infty
$$


and

$$
2^{-n} \operatorname{card}\left\{j: 2^{n}\left\|\triangle_{j}\right\|_{H} \geq \rho\right\} \leq \rho^{-1} \sum_{j=1}^{2^{n}}\left\|\triangle_{j}\right\|_{H} \leq \frac{C}{\rho} .
$$

However

$$
\int_{\left\{\left\|h_{n}\right\|_{H} \geq \rho\right\}}\left\|h_{n}\right\|_{H} d \lambda=\sum_{\left\{j: 2^{n}\left\|\triangle_{j}\right\|_{H} \geq \rho\right\}}\left\|\triangle_{j}\right\|_{H}
$$

Now, (B.9) follows from (B.10), (B.11) and the $\|\cdot\|_{H}$-absolute continuity of $\varphi$, (B.3). Consequently, by the definition of Bochner integral, for $0 \leq j<k \leq 2^{n}$,

$$
\int_{j / 2^{n}}^{k / 2^{n}} g(s) d s=\lim _{l \rightarrow \infty} \int_{j / 2^{n}}^{k / 2^{n}} h_{l}(s) d s=\varphi\left(\frac{k}{2^{n}}\right)-\varphi\left(\frac{j}{2^{n}}\right) .
$$

Recall that the Bochner integral $h(u)=\int_{0}^{u} g(s) d s:[0,1] \rightarrow \mathbf{E}$ is continuous (see Diestel and Uhl [21, Theorem II.2.4]), and therefore, by (B.12) and the continuity of $\varphi$, for $0 \leq s<t \leq 1$, we have

$$
\int_{s}^{t} g(s) d s=\varphi(t)-\varphi(s)
$$

In particular, for $t \in[0,1]$, we have

$$
\varphi(t)=\int_{0}^{t} g(s) d s
$$

Finally, we shall show that $b \geq \widetilde{\Lambda}(\varphi)$. Let $\Pi: 0=t_{0}<t_{1}<\cdots<t_{N}=1$ be a partition of $[0,1]$, where $\|\Pi\| \triangleq \max _{1 \leq i \leq N}\left|t_{i}-t_{i-1}\right|$ will be taken to be sufficiently small. Note that $\widetilde{\Lambda}\left(\varphi_{n}\right)=\sum_{i=1}^{N} \int_{t_{i-1}}^{t_{i}} \Lambda\left(g_{n}\right) d \lambda$. By Jensen's inequality,

$$
\int_{t_{i-1}}^{t_{i}} \Lambda\left(g_{n}\right) d \lambda \geq\left(t_{i}-t_{i-1}\right) \Lambda\left(\frac{1}{t_{i}-t_{i-1}} \int_{t_{i-1}}^{t_{i}} g_{n} d \lambda\right) \text {. }
$$

Therefore

$$
\widetilde{\Lambda}\left(\varphi_{n}\right) \geq \sum_{i=1}^{N}\left(t_{i}-t_{i-1}\right) \Lambda\left(\frac{\varphi_{n}\left(t_{i}\right)-\varphi_{n}\left(t_{i-1}\right)}{t_{i}-t_{i-1}}\right) .
$$

Using the lower semicontinuity of $\Lambda$, we obtain

$$
b=\liminf _{n \rightarrow \infty} \widetilde{\Lambda}\left(\varphi_{n}\right) \geq \sum_{i=1}^{N}\left(t_{i}-t_{i-1}\right) \Lambda\left(\frac{\varphi\left(t_{i}\right)-\varphi\left(t_{i-1}\right)}{t_{i}-t_{i-1}}\right)=\widetilde{\Lambda}\left(\varphi_{\Pi}\right)
$$

for any partition $\Pi$, where $\varphi_{\Pi}$ is defined as follows

$$
\varphi_{\Pi}(t)=\int_{0}^{t} g_{\Pi}(s) d s, \quad t \in[0,1],
$$

where

$$
g_{\Pi}(t)=\frac{\varphi\left(t_{i}\right)-\varphi\left(t_{i-1}\right)}{t_{i}-t_{i-1}} \quad \text { for } t \in\left[t_{i-1}, t_{i}\right)
$$

and $g_{\Pi}(1)=\varphi(1)$. 
Let $\Pi_{m}$ be a partition such that $\left\|\Pi_{m}\right\| \leq \frac{1}{m}$. Define

$$
g_{\Pi_{m}}(t)=\frac{\varphi\left(t_{i}^{(m)}\right)-\varphi\left(t_{i-1}^{(m)}\right)}{t_{i}^{(m)}-t_{i-1}^{(m)}} \quad \text { for } t \in\left[t_{i-1}^{(m)}, t_{i}^{(m)}\right)
$$

and $g_{\Pi_{m}}(1)=\varphi(1)$, where $\Pi_{m}: 0=t_{0}^{(m)}<t_{1}^{(m)}<\cdots<t_{m_{N}}^{(m)}=1$. Since $\int_{0}^{1}\|g(s)\|_{H} d s<\infty, \lim _{m \rightarrow \infty}\left\|g_{\Pi_{m}}-g\right\|_{H}=0$ a.e. Furthermore, $\lim _{m \rightarrow \infty}\left\|g_{\Pi_{m}}-g\right\|=$ 0 a.e. Consequently, taking account of the lower semicontinuity of $\Lambda$, we have $\liminf _{m \rightarrow \infty} \Lambda\left(g_{\Pi_{m}}\right) \geq \Lambda(g)$ a.e., which, as well as Fatou's lemma, implies

$$
\liminf _{m \rightarrow \infty} \int_{0}^{1} \Lambda\left(g_{\Pi_{m}}\right) d \lambda \geq \int_{0}^{1} \liminf _{m \rightarrow \infty} \Lambda\left(g_{\Pi_{m}}\right) d \lambda \geq \int_{0}^{1} \Lambda(g) d \lambda=\widetilde{\Lambda}(\varphi) .
$$

From (B.13) and (B.14) it follows that

$$
b \geq \liminf _{m \rightarrow \infty} \widetilde{\Lambda}\left(g_{\Pi_{m}}\right) \geq \widetilde{\Lambda}(\varphi),
$$

which proves the desired results. The lemma is proved.

Lemma B.3. For any $a>0, K_{a}=\{\varphi: \widetilde{\Lambda}(\varphi) \leq a\}$ is compact in $\left(C[0,1],\|\cdot\|_{\infty}\right)$.

Proof. Note first that: if $A \subset C([0,1] ; \mathbf{E})$ is such that

(i) There exist a compact set $K \subset \mathbf{E}$ such that $\varphi(t) \in K$ for all $t \in[0,1], \varphi \in A$;

(ii) $\lim _{\delta \rightarrow 0} \sup _{\varphi \in A} \omega_{\varphi}(\delta)=0$, where $\omega_{\varphi}(\delta)=\sup _{|t-s|<\delta}\|\varphi(t)-\varphi(s)\|$, then $\bar{A}$ is compact in $\left(C([0,1] ; \mathbf{E}),\|\cdot\|_{\infty}\right)$ (see also de Acosta [3] p. 88]).

Given $\varphi \in K_{a}$, let $g \in L^{1}([0,1], \mathbf{E})$ such that $\varphi(t)=\int_{0}^{t} g(s) d s$ for $t \in[0,1]$, then

$$
\|\varphi(t)-\varphi(s)\|=\left\|\int_{s}^{t} g(\tau) d \tau\right\| \leq \int_{s}^{t}\|g(\tau)\| d \tau
$$

which, together with Lemma B.1, yields (ii) for $A=K_{a}$.

Given $\varphi \in K_{a}$ again, let $g \in L^{1}([0,1], \mathbf{E})$ such that $\varphi(t)=\int_{0}^{t} g(s) d s$ for $t \in[0,1]$. For any $t \in(0,1]$, by Jensen's inequality

$$
\Lambda(\varphi(t))=t^{2} \Lambda\left(\frac{1}{t} \int_{0}^{t} g(s) d s\right) \leq t \int_{0}^{t} \Lambda(g(s)) d s \leq \widetilde{\Lambda}(\varphi) \leq a
$$

This proves (i) for $A=K_{a}$, with $K=\{x \in \mathbf{E} ; \Lambda(x) \leq a\}$, where $\{x \in \mathbf{E} ; \Lambda(x) \leq$ $a\}$ is compact in $(\mathbf{E},\|\cdot\|)$. Now the compactness of $K_{a}$ follows from the above arguments and the lower semicontinuity of $\widetilde{\Lambda}$ (Lemma B.2). The proof is completed.

From Lemma B.2 and Lemma B.3, we can obtain following theorem.

Theorem B.1. Let $\widetilde{\Lambda}$ be defined by (1.2). Under condition (1.4), $\widetilde{\Lambda}$ is a rate function on $\left(C([0,1] ; \mathbf{E}),\|\cdot\|_{\infty}\right)$.

\section{ACKNOWLEDGMENTS}

We would like to thank Professor Krzysztof Burdzy and Professor Amir Dembo for their comments. We are grateful to Professor Miguel A. Arcones for sending us his preprint. We are indebted to a referee who suggested Lemma 2.3 as well as the proof of Lemma 2.4, which led to the present improved version of the manuscript. 


\section{REFERENCES}

[1] de Acosta, A.: Upper bounds for large deviations of dependent random vectors, Z. Wahrsch. Verw. Gebiete 60, 551-565(1985). MR 87f:60036

[2] de Acosta, A.: Moderate deviations and associated Laplace approximations for sums of independent random vectors, Trans. Amer. Math. Soc. 329, 357-374(1992). MR 92e:60053

[3] de Acosta, A.: Large deviations for vector-valued Lévy processes, Stochastic Process. Appl. 51, 75-115 (1994). MR 96b:60060

[4] de Acosta, A.: Moderate deviations for empirical measures of Markov chains: lower bounds, Ann. Probab. 25, 259-284 (1997). MR 98f:60049

[5] de Acosta, A., Chen, Xia : Moderate deviations for empirical measures of Markov chains: upper bounds, J. Theoret. Probab. 11, 1075-1110 (1998) MR 99k:60069

[6] Araujo, A., Gine, E.: The Central Limit Theorem for Real and Banach Valued Random Variables, New York: Wiley, 1980. MR 83e:60003

[7] Arcones, M.A.: The large deviation principle for stochastic processes, Preprint, 2000.

[8] Billingsley, P.: Convergence of Probability Measures, New York: Wiley 1968. MR 38:1718

[9] Bolthausen, E.: Laplace approximations for sums of independent random vectors, Probab. Theory Related Fields 72, 305-318(1986). MR 88b:60075

[10] Borovkov, A. A.: Boundary value problems for random walks and large deviations in function spaces, Theory Probab. Appl. 12, 575-595(1967). (Russian original, MR 39:4906)

[11] Borovkov, A. A., Mogulskii, A. A.: Probabilities of large deviations in topological space I. Siberian Math. J. 19, 697-709(1978) (Russian original, MR 80c:60045)

[12] Borovkov, A. A., Mogulskii, A. A.: Probabilities of large durations in topological space II. Siberian Math. J. 21, 653-664(1980). (Russian original, MR 82c:60049)

[13] Chen, Xia: On the lower bound of the moderate deviations of i.i.d. random variables in a Banach space, Chinese Ann. Math. 11A, 621-629(1990). (in Chinese).

[14] Chen, Xia: Probabilities of moderate deviations for B-valued independent random vectors, Chinese J. Contemporary Math. 11, 381-393(1990).

[15] Chen, Xia: The moderate deviations of independent random vectors in a Banach space, Chinese J. Appl. Probab. and Statist. 7, 24-32(1991). MR 93m:60020

[16] Dembo, A., Zajic, T.: Large deviations: From empirical mean and measure to partial sums process, Stochastic Process. Appl. 57, 191-224(1995) MR 96m:60064

[17] Dembo, A., Zajic, T.: Uniform large and moderate deviations for functional empirical processes, Stochastic Process. Appl. 67, 195-211(1997) MR 98e:60013

[18] Dembo, A., Zeitouni, O.: Large Deviations Techniques and Applications, Boston: Jones and Bartlett, 1993. MR 95a:60034

[19] Deshayes, J., Picard, D.: Grandes et moyennes déviations pour le marches aleatoires, Astérisque 68, 53-71 (1979).

[20] Deuschel, J. D, Stroock, D. W.: Large Deviations, Boston: Academic Press 1989. MR 90h:60026

[21] Diestel, J., Uhl, J.: Vector Measures, American Mathematical Society, Providence, 1977. MR 56:12216

[22] Goodman, V., Kuelbs, J., Zinn, J.: Some results on the LIL in Banach space with applications to weighted empirical processes, Ann. Probab. 9, 713-752 (1981). MR 82m:60011

[23] Hu, Yijun: Large deviations for trajectories of sums of dependent random variables, Chinese J. of Contemporary Math, 19, 149-158(1998). MR 99i:60055

[24] Jiang, Tiefeng: Moderate deviations for double arrays with applications to moving average processes, Gaussian processes and Cesaro's sums, Preprint (1999).

[25] Karatzas, I., Shreve, S. E., Brownian Motion and Stochastic Calculus, 2nd ed., SpringerVerlag, 1991. MR 92h:60127

[26] Ledoux, M.: Sur les deviations moderees des sommes de variables a leatoires vectorielles independantes de meme loi, Probab. and Statist. Ann. Inst. Henri. Poincaré, 28, 267-280(1992). MR 93k:60017

[27] Ledoux, M., Talagrand, M.: Probability in Banach Spaces (Isoperimetry and Processes), Springer-Verlag, 1991. MR 93c:60001

[28] Lynch, J., Sethuraman, J.: Large deviations for processes with independent increments, Ann. Probab. 15, 610-627(1987). MR 88m:60076

[29] Mogulskii, A. A.: Large deviations in the space $C[0,1]$ for sums given on a finite Markov chain, Siberian Math. J. 15, 43-53(1974). (Russian original, MR 49:9914) 
[30] Mogulskii, A. A.: Large deviations for trajectories of multi-dimensional random walks, Theory Probab. Appl. 21, 300-315(1976). (Russian original, MR 54:8810)

[31] Schuette, P. H.: Large deviations for trajectories of sums of independent random variables, J. Theor. Probab. 7, 3-45(1994). MR 94m:60063

[32] Varadhan, S. R. S.: Asymptotic probabilities and differential equations, Comm. Pure Appl. Math. 19, 261-286(1966) MR 34:3083

[33] Wu, Liming: Some general methods of large deviations and applications, Preprint (1991), in $\langle\langle$ Habilitation a diriger des recherches $\rangle\rangle$, Laboratoire de Probabilités de Paris VI, 1993.

Department of Mathematics, Wuhan University, Wuhan, Hubei 430072, People's RePUBLIC OF CHINA

E-mail address: yijunhu@public.wh.hb.cn

Department of Mathematics, University of Maryland, College Park, Maryland 20742

E-mail address: tyl@math.umd.edu 